\title{
A systematic approach to illuminate a new hot spot of avian influenza circulation in South Vietnam
}

\author{
Kien Le ${ }^{1}$, Mark Stevenson ${ }^{2}$, Norikazu Isoda ${ }^{1}$, Huy Chu $^{3}$, Tien Nguyen ${ }^{3}$, Long Nguyen ${ }^{3}$, \\ Tien Tien ${ }^{3}$, Trung Le ${ }^{3}$, Keita Matsuno ${ }^{1}$, Masatoshi Okamatsu ${ }^{1}$, and Yoshihiro Sakoda ${ }^{1}$ \\ ${ }^{1}$ Hokkaido Daigaku Juigakubu Daigakuin Juigaku Kenkyuka \\ ${ }^{2}$ University of Melbourne \\ ${ }^{3}$ Ministry of Agriculture and Rural Development
}

April 8, 2021

\begin{abstract}
In South Vietnam, live bird markets (LBMs) are key in the value chain of poultry products and spread of avian influenza virus (AIV) although they may not be the sole factor to determine avian influenza (AI) prevalence in the southern part. Therefore, a risk analysis of AIV spread was conducted by including all possible value chain factors. A cross-sectional study was performed in backyard farms, high-biosecurity farms (bio-farms), LBMs, and poultry delivery stations (PDSs) in the four districts of Vinh Long Province in December 2016 and August 2017. A total of 3597 swab samples were collected from individual poultry at 101 backyard farms, 50 bio-farms, 58 sellers in LBMs, and 17 traders in PDSs and then investigated for AIV isolation. Concurrently, information related to participants and birds was collected and used to identify the fixed and random effects of factors in AIV infection. A total of 274 birds were positive for virus isolation, with a prevalence of $7.6 \%$ (95\% confidence interval [CI]: 6.8-8.5) at the individual poultry level, and the adjusted prevalence based on the sampling weight was $7.9 \%(95 \%$ CI: 7.6-8.2). The significantly higher prevalence in PDSs (20.7\%) and LBMs (14.2\%) compared to backyard farms (3.0\%) and bio-farms $(0.6 \%)$ suggested that PDSs are another hot spot for AIV circulation. The high diversity in the seller and trader population characteristics was revealed using multiple-correspondence analysis to analyze the participants' demographic factors in LBM and PDS. The mixed-effect logistic regression model revealed that keeping duck at the sampling time and the owner's older age should be risk factors of AIV infection in PDS. Therefore, functional AI control efforts to monitor the PDS system should be emphasized to minimize AIV circulation risk in Vietnam.
\end{abstract}

\section{Hosted file}

Manuscript_Le_et_al.pdf available at https://authorea.com/users/406470/articles/517126a-systematic-approach-to-illuminate-a-new-hot-spot-of-avian-influenza-circulation-insouth-vietnam 
Table 1. Unconditional associations between the outcome (virus isolation positive) and the four explanatoryvariables

\begin{tabular}{lllll}
\hline \multicolumn{1}{c}{ Variables } & AIV positive & No. of sample & Prevalence $(95 \%$ CI $)$ & P-value \\
\hline Sector & & & & \\
Backyard & 32 & 1,056 & $3(2.1-4.3)$ & Reference \\
Bio-farm & 7 & 1,200 & $0.6(0.2-1.2)$ & $<0.001$ \\
LBM & 94 & 660 & $14.2(11.7-17.1)$ & $<0.001$ \\
PDS & 141 & 681 & $20.7(17.7-23.9)$ & $<0.001$ \\
& & & & \\
Species sampling & & & & \\
Chicken & 100 & 1,801 & $5.6(4.5-6.7)$ & Reference \\
Duck & 157 & 1,575 & $10(8.5-11.6)$ & $<0.001$ \\
Muscovy duck & 16 & 189 & $8.5(4.9-13.4)$ & 0.106 \\
Environment & 0 & 18 & $0(0-18.5)$ & 0.97 \\
Goose & 1 & 14 & $7.1(0.2-33.9)$ & 0.266 \\
& & & & \\
Round & & & & \\
1 (2016) & 107 & 1,814 & $5.9(4.9-7.1)$ & Reference \\
2 (2017) & 167 & 1,783 & $9.4(8.1-10.8)$ & $<0.001$ \\
& & & & \\
District & & & & \\
Binh Minh & 61 & 910 & $6.7(5.2-8.5)$ & Reference \\
Long Ho & 61 & 909 & $6.7(5.2-8.5)$ & 0.843 \\
Mang Thit & 53 & 867 & $6.1(4.6-7.9)$ & 0.612 \\
Tam Binh & 99 & 911 & $10.9(8.9-13.1)$ & 0.004 \\
\hline
\end{tabular}




\begin{tabular}{|c|c|c|c|c|c|c|c|c|c|}
\hline Category & Population & $\begin{array}{c}\text { Selected for } \\
\text { sampling }\end{array}$ & $\begin{array}{l}\text { Likelihood of } \\
\text { being selected (\%) }\end{array}$ & $\begin{array}{l}\text { Sampling } \\
\text { weight }\end{array}$ & $\begin{array}{c}\text { AIV } \\
\text { positive }\end{array}$ & $\begin{array}{l}\text { No. of } \\
\text { sample }\end{array}$ & $\begin{array}{l}\text { The prevalence } \\
\text { (95\% CI) }\end{array}$ & $\begin{array}{l}\text { The overall } \\
\text { prevalence } \\
(95 \% \mathrm{Cl})\end{array}$ & $\begin{array}{l}\text { The adjusted } \\
\text { prevalence } \\
(95 \% \mathrm{Cl})\end{array}$ \\
\hline $\begin{array}{l}\text { Backyard } \\
\text { farm }\end{array}$ & 1,288 & 101 & 7.8 & 13 & 32 & 1,056 & $3(2.1-4.3)$ & & \\
\hline Bio-farm & 228 & 50 & 21.9 & 5 & 7 & 1,200 & $0.6(0.2-1.2)$ & $7.6(6.8-8.5)$ & $7.9(7.6-8.2)$ \\
\hline LBM & 123 & 12 & 9.8 & 10 & 94 & 660 & $14.2(11.7-17.1)$ & & \\
\hline PDS & 98 & 13 & 13.3 & 8 & 141 & 681 & $20.7(17.7-23.9)$ & & \\
\hline
\end{tabular}




\begin{tabular}{|c|c|c|c|c|}
\hline Variable & AIV positive & Bird & OR $(95 \% \mathrm{CI})$ & $\begin{array}{l}\begin{array}{l}\text { P-value } \\
\text { (ANOVA) }\end{array} \\
\end{array}$ \\
\hline \multicolumn{5}{|l|}{ District } \\
\hline Binh.Minh & 25 & 164 & Ref & \multirow[t]{4}{*}{$<0.001$} \\
\hline Long.Ho & 35 & 189 & $1.26(0.72-2.22)$ & \\
\hline Mang.Thit & 6 & 150 & $0.23(0.09-0.58)$ & \\
\hline Tam.Binh & 75 & 178 & $4.05(2.41-6.81)$ & \\
\hline \multicolumn{5}{|l|}{ Species } \\
\hline Chicken & 38 & 397 & Ref & \multirow[t]{5}{*}{$<0.001$} \\
\hline Duck & 103 & 196 & $10.46(6.76-16.19)$ & \\
\hline Environment & 0 & 18 & NA & \\
\hline Goose & 0 & 3 & NA & \\
\hline Muscovy duck & 0 & 67 & NA & \\
\hline \multicolumn{5}{|l|}{ Mix species } \\
\hline No & 2 & 40 & Ref & \multirow[t]{2}{*}{0.004} \\
\hline Yes & 139 & 641 & $5.26(1.25-22.08)$ & \\
\hline \multicolumn{5}{|l|}{ Gender } \\
\hline Female & 139 & 551 & Ref & \multirow[t]{2}{*}{$<0.001$} \\
\hline Male & 2 & 130 & $0.05(0.01-0.19)$ & \\
\hline \multicolumn{5}{|l|}{ Year old } \\
\hline $31-40$ & 0 & 90 & & \multirow[t]{4}{*}{$<0.001$} \\
\hline $41-50$ & 2 & 128 & Ref & \\
\hline $51-60$ & 138 & 348 & $41.4(10.07-170.13)$ & \\
\hline$>60$ & 1 & 115 & $0.55(0.05-6.18)$ & \\
\hline \multicolumn{5}{|c|}{ Career experience (year) } \\
\hline $1-5$ & 0 & 149 & NA & \multirow[t]{3}{*}{$<0.001$} \\
\hline $6-10$ & 25 & 94 & Ref & \\
\hline$>10$ & 116 & 438 & $0.99(0.6-1.65)$ & \\
\hline \multicolumn{5}{|l|}{ Selling poultry meat } \\
\hline No & 85 & 492 & Ref & \multirow[t]{2}{*}{$<0.001$} \\
\hline Yes & 56 & 189 & $2.02(1.36-2.98)$ & \\
\hline \multicolumn{5}{|l|}{ Working area } \\
\hline Within commune & 0 & 30 & NA & \multirow[t]{4}{*}{$<0.001$} \\
\hline Within district & 6 & 120 & Ref & \\
\hline Within province & 25 & 198 & $2.75(1.09-6.9)$ & \\
\hline Interprovincial & 110 & 333 & $9.37(4-21.97)$ & \\
\hline \multicolumn{5}{|l|}{ Working range (km) } \\
\hline$<10$ & 6 & 100 & Ref & \multirow[t]{5}{*}{$<0.001$} \\
\hline $10-50$ & 0 & 104 & $\mathrm{NA}$ & \\
\hline $51-100$ & 27 & 213 & $2.27(0.91-5.7)$ & \\
\hline $101-200$ & 0 & 75 & NA & \\
\hline $201-500$ & 108 & 189 & $20.89(8.72-50.07)$ & \\
\hline \multicolumn{5}{|c|}{ Frequency of poultry gathering } \\
\hline once per day & 33 & 492 & Ref & \multirow[t]{3}{*}{$<0.001$} \\
\hline twice per day & 41 & 92 & $11.18(6.5-19.23)$ & \\
\hline thrice per day & 67 & 97 & $31.06(17.8-54.22)$ & \\
\hline \multicolumn{5}{|l|}{ Flock size (head) } \\
\hline$<50$ & 6 & 179 & Ref & $<0.001$ \\
\hline $50-100$ & 2 & 40 & $1.52(0.29-7.81)$ & \\
\hline $101-500$ & 2 & 124 & $0.47(0.09-2.38)$ & \\
\hline $501-1000$ & 107 & 259 & $20.3(8.67-47.52)$ & \\
\hline$>1000$ & 24 & 79 & $12.58(4.89-32.36)$ & \\
\hline
\end{tabular}

\section{Hosted file}

Table_4.pdf available at https://authorea.com/users/406470/articles/517126-a-systematicapproach-to-illuminate-a-new-hot-spot-of-avian-influenza-circulation-in-south-vietnam 
Fig. 1.

Le et al.

a)

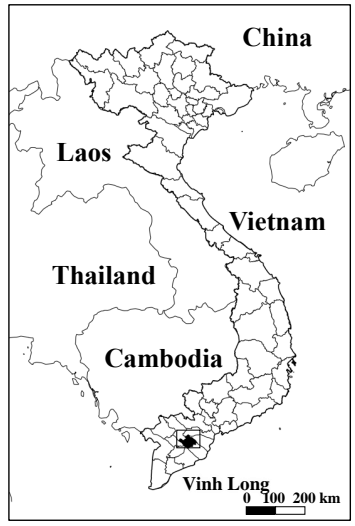

b)

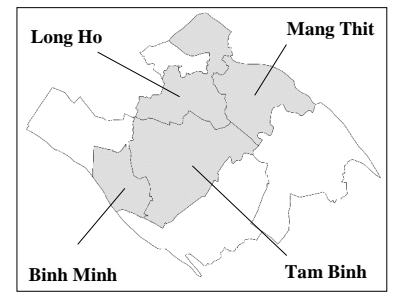


Fig. 2.

Le et al.
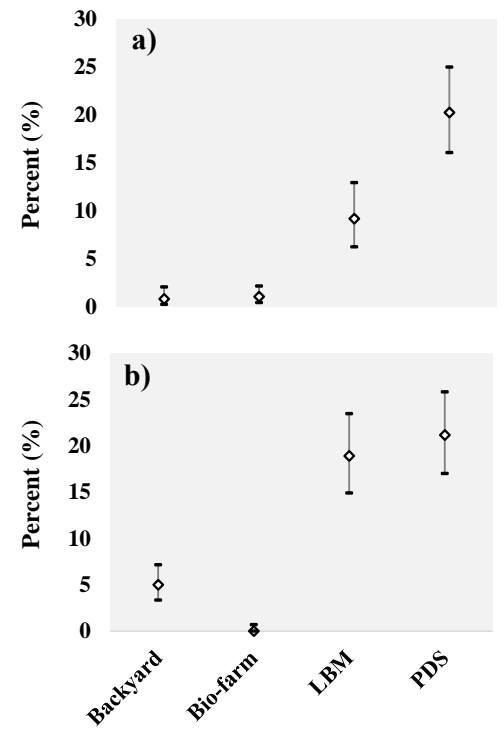
Fig. 3.

Le et al.
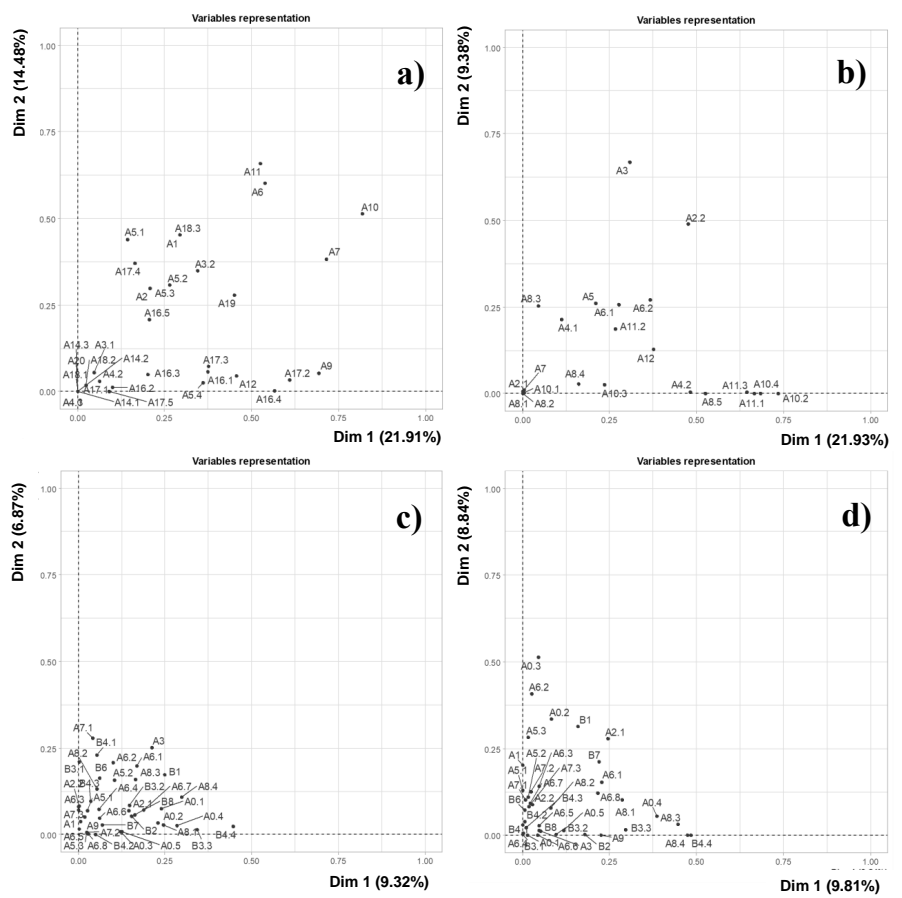
Fig. 4.

Le et al.
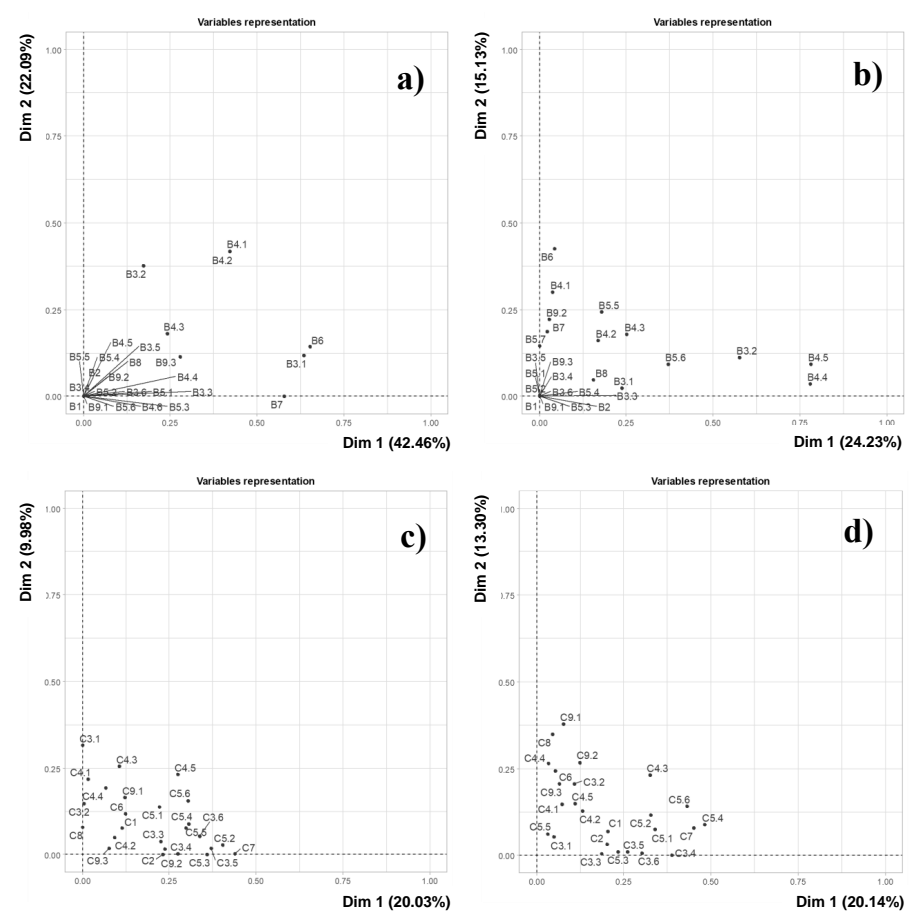
Fig. 5.

Le et al.
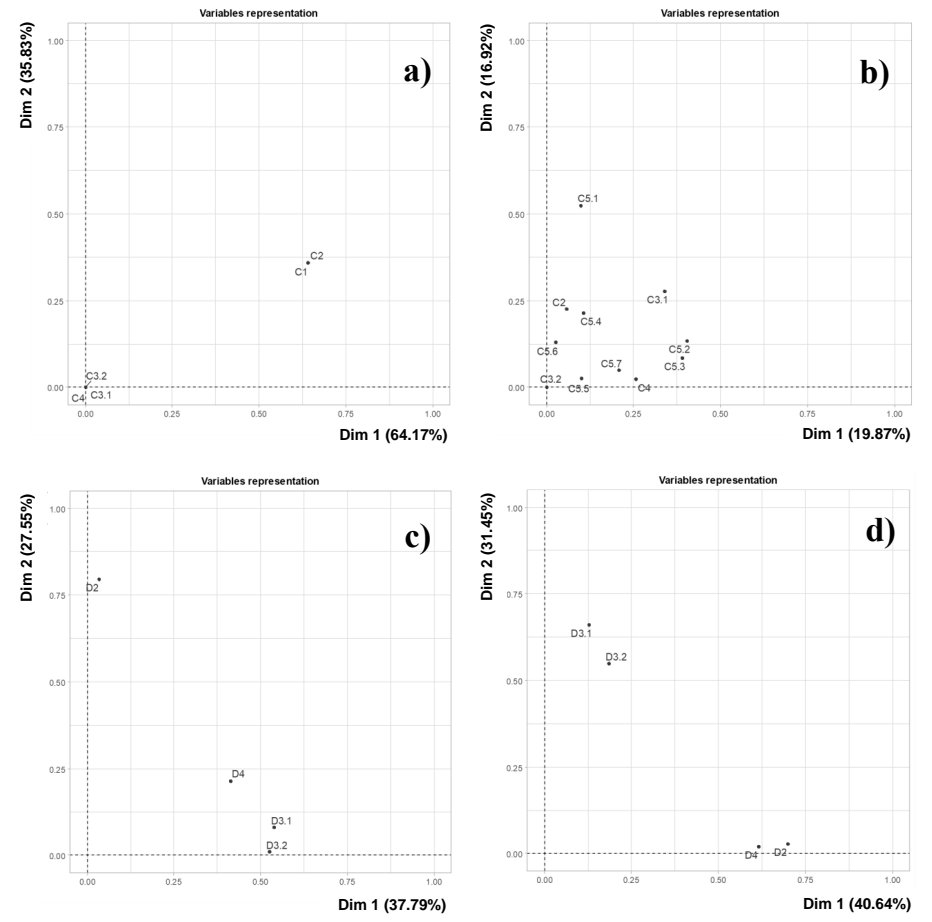
Fig. 6.

Le et al.
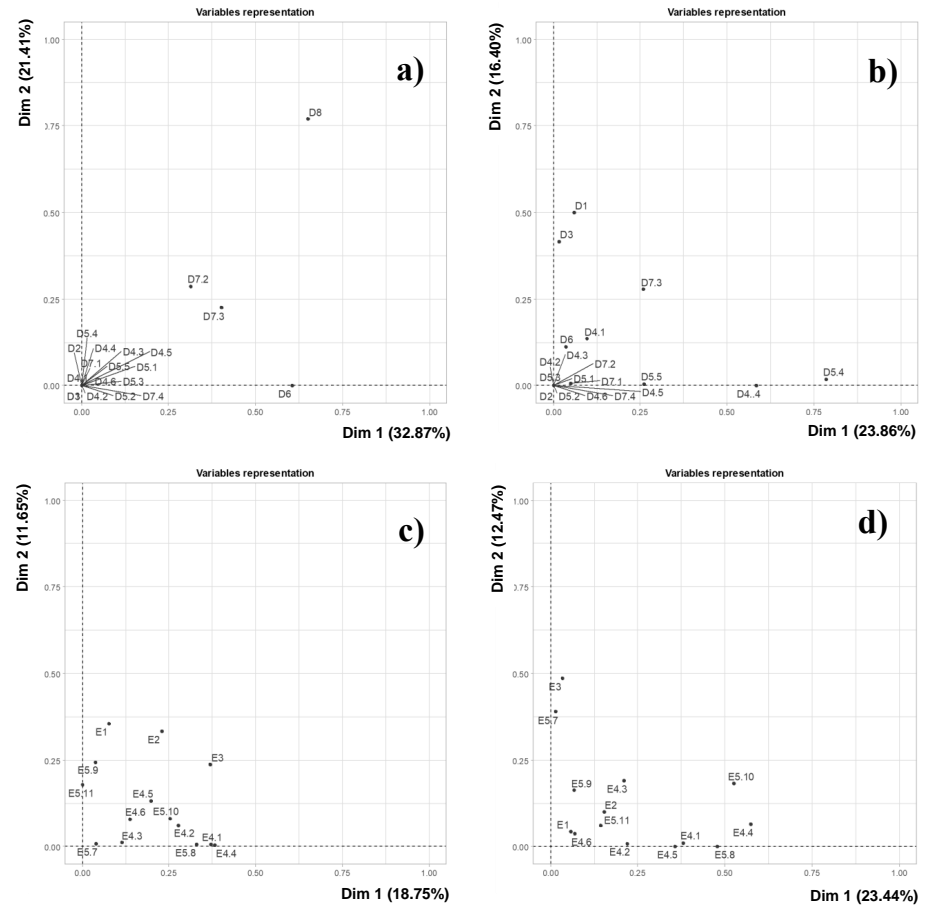